\title{
Visages et représentations de femmes dans les situations de conflit : l'exemple de Murambi le livre des ossements de Boris Boubacar Diop et d'Allah n'est pas obligé d'Ahmadou Kourouma
}

\author{
Flora Amabiamina \\ Université de Douala
}

\section{Introduction}

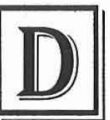

e la différence sexuelle, que nul ne peut nier aujourd'hui, découlent des rôles et conduites assignés aux deux genres qui sont loin d'être identiques. L'imaginaire collectif a, de tout temps, campé la femme et l'homme dans des caractères et des attitudes différents qui, non sans mal, continuent de conditionner l'appréhension des genres et les agissements humains dans le commerce ordinaire du quotidien ${ }^{1}$. Cela conduit inévitablement à l'établissement de stéréotypes consacrés telle la virilisation de la femme que l'on tient pour contre nature une fois qu'elle se démarque du statut posé comme sien " naturellement ". C'est le cas des femmes soldats ou de celles qui doivent se départir de leur nature première et faire face à des situations de conflit ou d'urgence et doivent, pour survivre, agir comme des hommes, à l'image de se battre en arborant, au

1 Virginia Woolf, le schématise dans ce qu'elle dénomme des "lignes de démarcation mystiques entre lesquelles sont fixés, rigides, séparés, artificiels, les êtres humains ", in Trois Guinées, trad. V. Forrester, Paris, Editions des femmes, 1977, p. 200.

2 Le roman de Boubacar Boris Diop s'inscrit dans une dynamique de réflexion sur le génocide de 1994 au Rwanda qui a mobilisé une dizaine d'écrivains. C'est à une sorte de " devoir de mémoire " qu'invitent alors ces auteurs parmi lesquels Tierno Monénembo, L'aîné des orphelins, Paris, Seuil, 2000, Koulsy Lamko, La phalène des collines, Ed., Kuljaama, 2000, Véronique Tadjo, $A$ l'ombre d'Imana, Arles, Actes Sud, 2000. Tous ces récits qui racontent les massacres, les horreurs du génocide dans des perspectives singulières, chaque auteur suivant son ressenti du génocide, accordent une place particulière à la condition de la femme. 
besoin, des tenues militaires et des armes. Murambi, le livre des ossements de Boris Boubacar Diop ${ }^{2}$ et Allah n'est pas obligé d'Ahmadou Kourouma ${ }^{3}$ nous en offrent des exemples éloquents. Les deux textes sont bâtis à partir d'histoires vraies de conflits ethniques; celle du génocide des Tutsi qui a eu lieu au Rwanda en 1994, pour le premier, et celle des enfants enrôlés dans les guerres tribales qui ont dévasté le Liberia et la Sierra Leone dans les années quatre-vingt-dix, pour le second.

Dans notre analyse les situations de conflit seront, non seulement, des états où la guerre est manifeste, comme dans le roman de Kourouma, mais aussi ceux-là où elle est latente, sourde, mais tout autant, sinon plus, pernicieuse que dans le premier cas, tel qu'il en est dans l'univers dépeint par Boubacar Diop. De prime abord, la présence de femmes dans des univers en conflit ne pourrait être rattachée, rationnellement, qu'à une fin décorative. Les femmes devraient y être de simples éléments ornementaux, des sortes de personnages figuratifs, qui serviraient à planter le décor, n'étant pas tenues comme des forces sur lesquelles on devrait compter, en premier, en telles situations. Tout au plus, en seraient-elles les premières victimes ${ }^{4}$.

$\mathrm{Au}$ vrai, on ne devrait pas retrouver la femme en première ligne, c'està-dire comme actrice des combats qui se déroulent, ou y jouant un rôle déterminant. Les conflits étant par essence des moments dangereux, la femme, l'être dit faible, devrait être protégée. Mais ce n'est pas l'option choisie par les écrivains dont les textes serviront dans cette analyse. Le fait est d'autant plus intéressant que ce sont des hommes qui campent les femmes dans des positions généralement réservées aux hommes. C'est l'homme qui est doté de courage, qui est censé faire face à de situations les plus difficiles, voire les plus cruelles. Loin d'être complaisants, ces auteurs montrent par la preuve de la fiction que l'histoire des sociétés ne peut s'écrire ni se construire sans la présence et la participation de la femme. La femme, davantage que son pair l'homme, fut-il encore besoin de le rappeler, est aux fondements de l'humanité, de par sa fonction parturiente. Et elle doit, pour cela même, s'impliquer dans son être et son devenir quelle que soit alors la situation qui prévaut. L'histoire des guerres et des révolutions qui ont causé de nombreux ravages dans le monde avec leur lot de résistantes, ces espèces de mères

3 Les titres Murambi le livre des ossements et Allah n'est pas obligé seront abrégés dans la suite des développements pour indiquer les pages que nous citons tel que suit et respectivement : MLLDO, ANPO.

4 Les guerres qui déchirent, ces temps derniers, les pays tels la République démocratique du Congo, le Soudan (Darfour), le Niger, la Pépublique centrafricaine, le montrent à suffisance. 
courage que l'on découvre de plus en plus, ces femmes qui ont forcé l'admiration des hommes de par leur audace et leur abnégation, montrent à escient qu'à défaut de porter des tenues de treillis et chausser des brodequins pour camper au devant des lignes de front, elles peuvent, comme leurs alter ego masculins, faire la guerre par différents moyens. On s'attachera ainsi, dans un premier temps, au type de femmes que l'on retrouve dans ces écrits en mettant l'accent sur leurs portraits à la fois physique, moral et psychique et, dans un second temps, on mettra en évidence les actions qu'elles posent et leurs incidences sur les logiques en présence en nous servant du schéma actantiel élaboré par Greimas.

\section{Portraits des femmes en présence dans les situations de conflit}

Nombre d'études réalisées, notamment sociologiques 5 , ces temps derniers sur les différentes guerres civiles qui ont lieu sur les terres africaines, s'attachent à mettre en relief le sort réservé aux femmes et aux enfants qui sont, généralement, les premières victimes des exactions perpétrées lors des guerres. Et si cela est ainsi, c'est bien parce qu'a priori ils sont considérés comme les plus vulnérables, ceux-là même que l'on doit protéger, comme la veuve et l'orphelin. Seulement, on observe également que loin de représenter des créatures en besoin de protection, elles figurent aussi souvent parmi les guerriers, ceux qui se battent ou alors comptent, parfois, parmi les bourreaux, ceux qui maltraitent. C'est le cas de celles que l'histoire et l'idéologie ont dénommé les amazones. L'image de l’amazone est élastique et évolue au fil du temps et ce depuis son émergence. Ici nous prendrons en compte tous les aspects qui lui ressortissent et qui s'entr'aperçoivent au travers des récits en établissant des portraits qui se veulent englobants réunissant, à la fois, des éléments physique et psychique.

\section{Des femmes Masculines}

Ce sont des femmes que l'on qualifie généralement de viriles ou encore de viragos; l'expression se retrouve d'ailleurs dans le roman d'Ahmadou

5 Les différents rapports des organisations internationales tels Amnesty International, Human Rigths Watch, l'ONU, ou encore l'essai de Bolya, $L a$ profanation des vagins - Le viol, arme de destruction massive, Paris, Editions du Rocher, 2005, s'arrêtent sur les violences, en l'occurrence sexuelles, faites aux femmes dans les guerres (Algérie, Bosnie, Côte d'Ivoire, République démocratique du Congo, Rwanda, Sierra Leone...). 
Kourouma (ANPO, 146). Elles se démarquent des autres, des féminines, parce qu'elles ressemblent par bien d'aspects autant physiques que caractériels à des hommes. Elles sont, à nombre d'égards, fidèles à l'image de l'amazone. Ce qui caractérise, en premier, ces femmes pas comme les autres c'est leur physique peu gracieux et profondément masculinisé d'où la dénomination d'hommes manqués qui leur est souvent associée. Leur physique figure, pour certaines, des présages des actions qu'elles assument dans le récit, on pourrait alors légitimement croire que leurs attributs physiques les prédisposent déjà à des missions singulières.

Il en est ainsi de Jessica dans Murambi le livre des ossements qui est doté d'un physique ingrat, " un corps sec et sans grâce » (MLLDO, 82). Son accoutrement, une grande chemise d'homme qu'elle porte négligemment, n'arrange pas les choses. Elle ne fait aucun effort pour paraître féminine et se soucie peu de son apparence; seule la mission qu'elle s'est assignée, faire en sorte qu'il n'y ait plus jamais de génocide dans son pays, l'intéresse. D'avoir été entouré et d'avoir évolué longtemps parmi les hommes y est certainement pour quelque chose. Il est vrai qu'elle n'est présentée qu’à la fin de l'action car le génocide a déjà eu lieu. Mais on peut aussi, en toute logique, croire que ce physique, elle l'a depuis sa prime enfance. Elle est la préférée de son père, Jonas Sibomana, un ancien maquisard qui a côtoyé des chefs de guerre légendaires comme Che Guevara et Kabila. Du propre aveu de Jonas, de tous ses enfants Jessica est celle qui lui « ressemble le plus » (MLLDO, 43). Et si l'on s'arrête, un moment, sur ce personnage que le narrateur qualifie de " drôle de bonhomme " (MLLDO, Ib.), on peut penser que pour que Jessica soit tenue pour son sosie c'est qu'elle a dû être, très tôt, une espèce de garçon manqué. Jonas « avait un torse labouré de cicatrices et promettait de léguer tous ses biens à celui [de ses enfants] qui réussirait à reproduire sur son corps les mêmes blessures » (MLLDO, Id.). C'est à Jessica et non pas à un de ses fils qu'il lègue ses armes lorsqu'il songe qu'il est temps de passer l'arme, geste qui détermina sa fille à s'enrôler dans la guérilla aux côtés des forces du FPR.

Elle-même semble se plaire dans sa stature masculine qu'elle cultive, par ailleurs. Ses amis ne la considèrent-ils pas comme « une femme de caractère, du genre à faire peur aux hommes " (MLDO, 91)? Elle fume beaucoup, une habitude qu'elle a acquise pendant les négociations à Arusha. Jessica " conduit très bien " (MLLDO, 93); et l'on n'est pas sans ignorer que l'imaginaire collectif, quelle que soit la civilisation à travers un poncif désormais consacré, veut que les femmes soient de mauvaises conductrices. Elle est indépendante et libre. Elle avoue à son ami, 
Cornélius, qui s'inquiète de savoir si elle trouve le temps de s'occuper de sa vie sentimentale, qu'elle aime le sexe : "J'aime sentir un homme bouger dans mon corps. Ca fait du bien " (MLLDO, 92). Il convient de souligner qu'un des caractères attachés à l'image de l'amazone est la nymphomanie. Pour Jessica, il n'est nullement question de sentiment. D'ailleurs, elle n'a pas de compagnon. Il s'agit de bestialité, d'instinct à assouvir; et comme tel, tout homme ferait l'affaire. La preuve est qu'elle exprime ce besoin dans un langage peu sensuel. Il faut relever l'utilisation de l'article indéfini " un " qui, ici, marque l'indétermination. C'est dire qu'elle jetterait son dévolu sur n'importe quel homme du moment que ça en est un qui sache, bien sûr, " bouger ". Ainsi, on retrouve chez ce personnage une attitude mâle, virile et conquérante. C'est l'homme qui, d'ordinaire, s'offre le luxe de choisir ses partenaires et Jessica s'arroge ce droit, ce privilège de décider de qui elle veut, et de quand elle le veut.

Chez Kourouma, on ne retrouve pas une, comme il en est chez Boubacar Boris Diop, mais plusieurs femmes masculines à l'exemple de la mère supérieure, Marie Béatrice. Elle ressemble tant à un homme que le narrateur par le biais de l'adverbe évaluatif " tellement " invite à s'arrêter sur son apparence physique. Il la trouve, tellement, non seulement masculine, puisqu'il affirme qu'il était incongru de l'imaginer en train de faire l'amour avec un homme, mais aussi laide. Il suggère même implicitement que, pour un homme, avoir un acte sexuel avec une femme telle la nonne n'était pas loin de ressembler à une relation homosexuelle :

" On s'imaginait mal la sainte sous un homme en train de recevoir l'amour tellement elle était virago. [...] Elle était vraiment solide et de trop grande taille. Elle avait le nez largement étendu, les lèvres trop épaisses et les arcades sourcilières d'un gorille. Et puis elle avait la chevelure coupée ras. Et puis elle avait l'occiput plein de bourrelets comme chez les hommes. » (ANPO, 146)

Par le biais des analogies et des comparaisons que le narrateur opère, la masculinisation du personnage s'établit nettement; elle figure une hommasse. Du fait de la thériomorphisation générée par la caractérisation descriptive, elle sort du règne humain pour rejoindre celui de l'animal. Sa tenue, une soutane sur laquelle pendait un kalachnikov, vient en rajouter à la masculinisation de ce personnage. Et cette tendance à poser ces femmes comme des êtres en marge de la nature est marquée dans le roman de Kourouma qui en appelle à s'interroger sur ce travestissement. En effet, 
comment expliquer que les femmes d'ordinaire garantes de l'ordre social en arrivent à participer ou à perpétuer le désordre, à instaurer un climat de violence?

C'est de semblables interrogations qui transparaissent également dans la mise en scène des enfants-soldats féminins à peine sorties de l'adolescence. Si pour la plupart elles font les frais des violences, elles sont aussi celles-là qui sèment la terreur. Et dans ces cas-là, ce qui nous est donné à voir c'est leur côté terrifiant :

"Parmi [les] enfants-soldats, il y a des filles, oui des vraies filles qui ont le kalach, qui font le faro avec le kalach. Elles ne sont pas nombreuses. C'est aussi les plus cruelles; ça peut te mettre une abeille vivante dans ton œil ouvert » (ANPO, 56)

Birahima, l'enfant-soldat qui nous fait découvrir l'univers d'Allah n'est pas obligé l'apprend à ses dépens lorsqu'il tombe dans une embuscade tendue par d'autres enfants-soldats sur sa route pour le Liberia. Le premier soldat auquel il fait face est une fille qu'il masculinise et chosifie :

"Nous avons vu apparaître un enfant-soldat. Un small soldier, c'était pas plus haut que le stick d'un officier. Un enfant-soldat en tenue de parachutiste beaucoup trop grande. C'était une fille. Ça sortait d'un pas hésitant. Et puis ça a regardé le travail accompli par la mitraille, examiné comme si un mec pouvait se relever alors que tout le monde était mort et même le sang était fatigué de couler. Il s'est arrêté et puis ça a sifflé et resifflé fort. Et de partout ont débouché des enfants-soldats, tous habillés pareil que le premier, tous faisant le faro avec le kalach " (ANPO, 58)

Le soldat-fille ne semble pas être une fille au regard de la présentation qu'en fait le narrateur. De fait, seule son appartenance à la classe des guerriers l'honore; autrement, c'est une chose. Dans la présentation préambulaire, le lecteur est mis en face d'un enfant-soldat, certes, qui pourrait être de tout sexe puisque c'est l'article indéfini « un » qui est repris par trois fois pour le présenter. Seulement, ce procédé d'insistance n'est pas gratuit. On pourrait croire que c'est un effet ménagé par le narrateur pour préparer à la surprise que lui-même, par ailleurs, ne manque pas d'éprouver à sa découverte : "c'était une fille ». Ne la désigne-t-il pas par les pronoms démonstratifs "ça " et " c' " qui, dans ce cadre, revêtent, à n'en pas douter, une valeur péjorative? Le pronom personnel masculin " il " et le déterminant masculin « le » mobilisés dans cette description du personnage 
contribuent également à sa virilisation. Il n'y a manifestement pas de mise à distance du narrateur dans cette séquence ${ }^{6}$.

En réalité, on peut percevoir l'indignation de ce narrateur qui n'admet pas ce renversement des valeurs. Non seulement le jeune soldat est une enfant mais, pire encore, c'est une fille! D'ailleurs, on remarque que ce ne sont que les soldats de sexe féminin dont le genre est spécifiquement souligné tout le long du récit, traduisant par là même que le statut de guerrier est typiquement masculin, donc d'essence virile. Et l'on pourrait croire alors que c'est la " raison " qui fait en sorte que le narrateur caricature ces femmes qui sont sorties des stéréotypes et des « lignes de démarcation mystiques " dessinés par l'imaginaire collectif. Ainsi, les filles soldats dans Allah n'est pas obligé sont toutes désignées par le déictique " ça " qui les chosifie, et les attitudes dont elles sont affublées en rajoutent à cette caricature. Comme Jessica, dans Murambi le livre des ossements, elles fument. Si dans le roman de Boubacar Boris Diop la nature du stupéfiant n'est pas spécifiée (il pourrait s'agir du tabac), chez Kourouma, le narrateur nous signale qu'il s'agit de drogues dures, du hasch et de l'herbe que les filles soldats comme Sarah "[fument] et [croquent] sans discontinuer " (ANPO, 92); ce qui n'est pas sans effet sur leur état mental ${ }^{7}$.

Dans le roman de Kourouma, la caractéristique qui ressortit, en premier, aux femmes campées dans le rôle de soldats c'est la méchanceté. Et il n'est pas inutile de souligner que les stéréotypes caractériels reconnaissent à l'homme le monopole de la méchanceté et de la cruauté ${ }^{8}$.

6 Mais il est indéniable que si l'on tient compte du niveau de langue qui caractérise ce roman, on peut légitimement lire cette mobilisation de démonstratifs dans une autre perspective. Le héros narrateur Birahima a fait son éducation, quasiment seul, en dehors du système éducatif institutionnalisé, ce qui pourrait expliquer cette indifférenciation dans l'utilisation des déictiques.

7 Bolya dans Afrique, le maillon faible, Paris, Ed. Le Serpent à plumes, 2002, a montré, certes dans une argumentation très partisane, le "rôle considérable " que jouent les stupéfiants dans les guerres civiles de par le monde en s'attachant particulièrement au cas des enfants-soldats, « les jeunes guerriers du vide ", dont l'enrôlement a participé à l'accroissement de la consommation des drogues. Birahima, l'enfant-soldat et narrateur dans Allah n'est pas obligé le confirme lorsqu'il avoue " nous étions tous forts par le hasch comme des taureaux » (p. 119).

8 Ruth Amossy, dans Les idées reçues; Sémiologie du stéréotype, Paris, Nathan, 1991, p. 170, en dresse un tableau, reprenant les croyances stéréotypées de psychologues américains. 
Que ce soient les filles-soldats ou les femmes-soldats, elles sont presque toutes "méchantes, trop méchantes» (ANPO, 98). Ce n’est pas le cas de Jessica dans Murambi le livre des ossements qui est plutôt une femme de cour même si elle est dotée d'un caractère fort. Dans Allah n'est pas obligé, l'exemple le plus éloquent de la cruauté dont peuvent faire preuve les femmes dans des contextes graves est celui du général Onika Baclay Doe qui est à la tête d'une armée à elle confiée par son frère, Samuel Doe. Cette petite femme présentée comme "énergique comme un cabri auquel on a pris le petit » (ANPO, 113) n'a d'égard pour personne et surtout pas pour la valeur humaine, si ce n'est pour l'argent qu'elle se charge de faire fructifier par divers moyens illégaux. C'est une sorte d'ogresse. Ainsi lorsqu'elle est surprise s'apitoyant sur ses soldats morts sous les balles ennemies le narrateur ironise car, à ses yeux, la scène est des plus incongrues. L'utilisation du pronom démonstratif « ça " renforce cette ironie :

"Il fallait voir ça. Ça valait le déplacement. Une criminelle comme Onika pleurer sur des morts. Des larmes de crocodile! Ça ne pleurait pas sur les cadavres, mais sur ce que ça risquait de lui faire perdre " (ANPO, 118).

Le narrateur souligne, en outre, qu'elle " avait droit de vie et de mort sur tout le monde à Saniquellie [son territoire] et elle en usait. Et en abusait " (ANPO, 115). Elle y a instauré un code pénal et une justice qui lui sont propres. Tous les prévenus sont, d'emblée, coupables et " toujours condamnés à mort. Et les condamnés passent illico presto sur l'aire de l'exécution "(ANPO, 112). Il faut dire que son grade de "général " d'armée et sa qualité de sour du chef de l'État lui conferent, quelque peu, tous les droits. D'ailleurs, elle ne lui rend aucun compte et a créé un État dans l'État avec une gestion familiale. Mais, en réalité, on peut voir dans son organisation un exemple de société matriarcale, une société d'amazones. En effet, les postes les plus importants sont occupés par des femmes qui sont dotées, par là même, d'un pouvoir décisionnaire.

Ses trois brus ont été érigées au rang de commandants et leurs époux campent dans le rôle de figurants car ils ne sont que des personnages racontés. Le silence qui est fait autour de leur physique peut laisser entendre que s'il n'est pas insignifiant, il ne retient pas le regard. Ses femmes n'ont d'importance que par rapport aux rôles qu'elles assument. Elles sont en charge des finances (Sita Baclay), des prisons (Monita Baclay) 
et des enfants-soldats (Rita Baclay). Sita, la musulmane pratiquante, n'est pas « humanitaire pour un sou » (ANPO, 114); elle s'amuse du spectacle des hommes qu'elle fait fusiller. Rita, quant à elle, est une pédophile qui abuse des enfants dont elle s'occupe pour assouvir ses instincts pervers. Seule Monita, la protestante, fait preuve d'humanisme dans ce lot. Le narrateur la pare, d'ailleurs, d'" un cœur d'or » (ANPO, 114). Elle fait fi des ordres du général Onika et donne à manger aux prévenus affamés. Elle en fait même plus que son devoir ne l'y oblige en s'offrant en partage :

"Elle a donné du plaisir à ceux à qui il ne restait que quelques heures à vivre. Des gestes comme ça, Allah les voit et les récompense au ciel " (ANPO, 114).

Même si le narrateur ironise sur ce fait, on peut dire qu'elle fait partie de ces femmes qui, par delà le courage qui les caractérise, sont généreuses et prêtes à donner de leur personne pour réaliser leurs projets.

\section{Des feMmes DE CCEUR}

Si les situations font en sorte qu'elles doivent faire face, en se dépouillant de leur nature première, parce qu'elles n'ont pas souvent d'autre choix que celui-là, on se rend aussi compte que cette nature reprend souvent le dessus. Et c'est cette même nature qui les oblige, en quelque sorte, à se jeter dans les arènes pour mener des combats qui leur sont chers. Aussi les physiques ingrats dont certaines viragos sont affublées sont compensés par des cœurs en or. Et ce sont ces cœurs en or qui font paraître dans toute leur grandeur leur abnégation, leur humanisme.

Influencée très tôt, à 18 ans, par les activités de son père, Jessica s'est décidée à répondre à l'interpellation générée par les évènements de 1959 (le premier génocide des Tutsi) en rejoignant la guérilla à Mulindi, bravant tous les risques encourus. Elle a répondu à une nécessité, à un devoir. À Cornélius qui n'arrive pas à s'expliquer ses activités, elle répond par une question à laquelle elle apporte, elle-même, une réponse, mettant en relief l'opportunité de la chose :

"Faut-il attendre les sueurs les bras croisés ou tenter de faire quelque chose pour que notre pays redevienne normal? [...] Vous ne pouvez ne pas en tenir compte. La tragédie finit toujours par vous rattraper " (MLLDO, 45) 
Et justement pour ne pas se laisser engloutir par la tragédie, il faut s'engager et cela même au prix de sa vie. La crainte de mourir, elle l'a dépassée. Elle estime que " ce serait aujourd'hui pour quelqu'un comme [elle] presque une faute de goût [car sa vie à elle] ne vaut pas mieux que celle des milliers de gens qui tombent chaque jour " (MLLDO, 117). C'est pourquoi elle en arrive à s'interroger sur le droit qu' elle pourrait s'arroger de juger celles (ses consœurs, des Tutsi) qui, croyant échapper à la mort, ont choisi de pactiser avec l'ennemi : "Qui me donne, à moi Jessica, le droit de juger? Je ne sais pas ce que j'aurais fait à leur place » (MLLDO, 121). Elle se surprend à douter d'elle-même car, du même coup, elle réalise que, au vrai, l'acte désespéré de ces femmes n'est tout simplement que l'expression primaire de l'instinct de survie qui habite tout homme. Aussi, la victoire prochaine que lui prédit la belle inconnue, qui vient se confier à elle pour ne pas mourir l'esprit encombré d'horreurs ${ }^{9}$, elle la lui dédie : "Ce sera aussi ta victoire " (MLLDO, 123). Et par delà elle, c'est, en premier, à toutes les autres femmes, âmes torturées et violées qui ont désiré par dessus tout vivre, que cette victoire chèrement acquise appartient avant d'être celle de toute la communauté.

Jessica fait partie de ces martyrs au mental d'acier dont accouchent les conflits tribaux ou patriotiques. Même ayant vécu l'horreur et en disant l'inénarrable, elle trouve le moyen de plaisanter. De fait, elle a conservé un peu de l'innocence qui habite les enfants, cette innocence qui fait qu'elle peut perdre son sérieux lorsque la situation l'exige. C'est le cas lorsque, de retour au pays, leur ami Cornélius dont le père est surnommé le boucher de Murambi, en raison des massacres qu'il y a organisés, se retrouve avec eux et que la gêne s'installe. Elle parvient à détendre l'atmosphère sans que Cornélius, encore ignorant des forfaits de son père, ne se doute de quelque chose. L'humour c'est ce qui permet d'atténuer le tragique, c'est une sorte d'exutoire puisqu'il faut " vivre par-dessus tout et avec » (MLLDO, 85). Ce qui compte à ses yeux, c'est de se reconstruire alors que les apparences et même son histoire semblent lui dire tout le contraire. Son combat c'est de faire en sorte que la situation redevienne normale dans son pays, c'est-àdire qu'il recouvre la paix. Le vieux et sage Siméon ne s'y trompe pas alors lorsqu'il voit en elle « le genre de personne dont le Rwanda a besoin pour

9 La mort prend, chez la belle inconnue, une dimension particulière. C'est une forme d'ascèse, son témoignage est une sorte d'exorcisme. Bien que Jessica se refuse à écouter les détails scabreux de sa relation avec le prêtre, elle insiste pour les lui conter car dit-elle, " je ne veux pas mourir avec cela " (MLLDO, 122). 
se réconcilier avec lui-même » (MLLDO, 217), c'est dire la grandeur du personnage.

Cette abnégation et ce sens du sacrifice on les retrouve chez d'autres personnages féminins dans Murambi le livre des ossements. La religieuse Hutu, Félicité Niyutegeka, la "femme indomptable » (MLLDO, 141), qui paiera de sa vie son souci et son entêtement à sauver des vies des Tutsi, geste ô combien grand alors que l'heure est plutôt à la livraison des proies Tutsi. Une lecture en surface pourrait conclure que c'est sa qualité de femme au service de Dieu qui lui commande ce sacrifice; mais il n'en est rien car, à côté d'elle, d'autres personnages, des hommes d'église, ont joué le jeu des politiques Hutu qui invitaient et veillaient ${ }^{10}$ à l'extermination de ceux qu'ils dénomment les " cancrelats " (MLLDO, 26). Il en est ainsi du prêtre libidineux, que tout le monde connaît mais que nul ne veut nommer ${ }^{11}$, qui a fait croire aux femmes Tutsi qu'il pouvait leur sauver la vie, si elles consentaient à lui octroyer leurs faveurs. Autant la religieuse qui a sauvé des Tutsi de la mort a une identité nominale, autant l'homme de Dieu qui en a livré est recouvert d'anonymat, de ce point de vue. Il n'est désigné que par le nom générique "prêtre ». Cette caractérisation résonne comme une accusation. Ce personnage que la qualité pose, a priori, comme charitable ne l'est nullement; il rejoint, de fait, la bande d'égorgeurs sans foi ni loi. La sœur Félicité, à l'opposé, défie l'autorité et son frère qui la conjure de renoncer à ses activités (faire passer la frontière du Zaïre aux Tutsi pourchassés) pour embrasser la mort sans crainte. La lettre qu'elle laisse derrière elle est profonde et invite à lire la bêtise humaine. Son geste de cœur est de ceux qui veulent vaincre la haine et proclamer qu'il n'y a rien qui puisse surpasser la valeur humaine.

C'est aussi ce que l'on peut lire dans Allah n'est pas obligé à travers l'expérience des sœurs Marie Béatrice et Aminata qui vont jusqu’à transgresser la morale religieuse (elles violent le cinquième commandement qui veut que nul ne doit tuer) pour défendre leurs territoires et les âmes qui y vivent. La première devient soldat à la faveur des évènements, déterminée

10 Le préfet, au lendemain du début des massacres à l'église de Nyamata, vient s'assurer du bon déroulement des opérations et s'indigne de ce qu'il lui a été rapporté que dans l'église on a découvert quatre Tutsi " qui faisaient semblant d'être morts " (MLLDO, 109) ce qui, à ses yeux, est " trop " « MLLDO, Ib.).

11 On l'observe nettement dans l'échange que Jessica a avec la belle inconnue : " Je veux vous dire que j’ai couché avec ce prêtre. [...] En fait, je savais de qui il s'agissait. À Kigali, en ces jours de folie, tout le monde savait " (MLLDO, 118). 
qu'elle est de protéger son institution abritant de jeunes pensionnaires féminins contre vents et marées. Et pour cela, elle n'hésite pas à affronter le redoutable Prince Johnson. Sour Gabrielle Aminata, elle aussi, est responsable d'un pensionnat de jeunes filles qu'elle éduque et prépare au mariage. Dans ces univers où la guerre sévit, elles font preuve de charité en accueillant ces jeunes personnes souvent abandonnées à elles-mêmes de sorte qu'elles ne soient pas enrôlées dans des troupes armées ou contraintes de se prostituer pour survivre. Elles leur donnent une chance de grandir dans leur condition d'enfants en leur offrant un toit, le gîte et le couvert, avec en prime une éducation. Leur sens du sacrifice est grand car elles en arrivent à s'adjurer pour protéger leurs ouailles. Seul compte la survie de ces filles. Et pour mener à bien cette tache, prêcher la bonne parole et faire preuve de bons sentiments ne représentent pas toujours les meilleures armes et elles l'ont compris. Le climat l'exigeant, elles deviennent de véritables soldats qui maîtrisent l'art de la guerre comme des combattants aguerris. De fait, ces femmes se substituent aux hommes et assument les charges qui ont, depuis la nuit des temps, été dévolues à ces derniers, et auxquelles ils se dérobent à savoir, en premier, protéger les faibles et les nécessiteux.

Toutes ces femmes peuvent être qualifiées de véritables humanistes, tout simplement, parce qu'elles se révoltent devant l'intolérable misère, dans un univers hostile, et affrontent parallèlement les injustices faites aux plus démunis. Et ce faisant, elles brisent les tabous en essayant de construire un monde nouveau. On les retrouve ainsi dans le rôle d'agents.

\section{Des femmes en action : la typologie actantielle des femmes en récit}

Nous nous aiderons du schéma actantiel élaboré par Greimas pour arriver à déterminer les rôles et les places que tiennent les femmes dans ces univers décrits dans les récits et qui donnent à voir des situations de guerre ou de conflit. Nous nous attacherons, dans cette optique, à leur implication dans les actions qui prennent place. Cette typologie se veut totale parce que retenant tous les personnages féminins en présence dans les diégèses, qu'elles participent directement ou non aux actions du moment qu'elles contribuent à l'évolution de l'histoire de par leur seule présence. Si l'on admet, dans ce cadre, le principe suivant lequel les femmes représenteraient, au premier abord, des personnages de figuration, elles devraient être ce que Greimas dénomme des patients, c'est-à-dire des personnages qui subissent l'action. Mais, comme nous l'avons déjà

\section{4 - LittéRéalité}


souligné, ce n'est pas toujours le cas car elles endossent aussi la qualité d'agents, de celles qui sont à l'initiative d'actes soit en action soit en réaction. C'est parmi ces dernières qu'il convient de classer celles qui servent aussi " d'améliorateurs " ou de " dégradateurs " ${ }^{12}$ en qualité qu'adjuvants, celles qui aident à la quête d'un objet, ou d'opposants, celles qui nuisent à l'accomplissement de la quête de l'objet.

\section{DeS PROIES TOUTES INDIQUÉES : LES PATIENTS}

Les personnages considérés comme patients sont des actants qui sont affectés, suivant Claude Bremond, d'une manière ou d'une autre par les évènements racontés. Il en est ainsi des différents personnages que nous évoquerons dans ces analyses et qui subissent une modification de leur état à leurs dépens. C'est ce que nous soulignions déjà, lorsque nous posions les femmes comme les premières victimes des exactions perpétrées dans des situations de conflit. Elles sont des proies de premier ordre qui, résignées pour la plupart, attendent, pour certaines stoïquement, la mort suspendue sur leurs têtes comme l'épée de Damoclès.

Dans Murambi le livre des ossements, elles sont nombreuses ces femmes qui subissent les méfaits du génocide. Pour la plupart, elles se savent condamnées. Certaines " enduisent leur cou d'huile avant d'aller au lit. Elles disent : comme ça, quand les égorgeurs viendront, la lame de leur couteau fera moins mal " (MLLDO, 20). Il y a celles qui se retrouvent esseulées, leurs maris et enfants ayant été assassinés. Il en est ainsi de la jeune femme en noir qui hante les locaux de l'ancienne École technique pour prier inlassablement devant les squelettes emmêlés d'un homme enserrant un enfant, qu'elle a cru reconnaître comme étant les restes de son époux et de son enfant. Elle a manifestement du mal à se reconstruire car sa vie se réduit, désormais, à ce rituel. Et il y a aussi toutes ces femmes Tutsi, vieilles, jeunes ou nubiles, belles ou laides, qui subissent des viols innommables. Certaines, pour échapper à cette violence indicible, se livrent comme des agneaux de sacrifice à des bourreaux qui leur font miroiter un salut hypothétique voire improbable. La jeune fille, sans nom dans le récit, dont le seul crime est d'être née parmi les Tutsi et d'être de surcroît belle « comme le soleil » (MLLDO, 119), l'a appris à ses dépens

12 Les notions d' " améliorateur " et de " dégradateur » sont utilisées par Claude Bremond dans Logique du récit, Paris, Seuil, 1973, pour désigner les rôles d'actants qui entretiennent une relation de contiguïté avec les notions d'adjuvants et d'opposant dans la typologie actantielle de Greimas. 
après s'être donnée à l'homme d'église libidineux qui, à la seule fin d'assouvir ses instincts pervers, promettait de lui garantir la vie sauve. De nombreuses Tutsi crédules, dont l'instinct primaire de survie s'exprimait par dessus tout dans ces instants tragiques, ont connu le même sort. Il s'agit, dans le cas d'espèce, de viol.

Les viols en eux-mêmes sont déjà des actes odieux, des crimes. Et les circonstances dans lesquelles ils sont commis en rajoutent à l'horreur dont ils sont empreints. Par exemple, parmi les violeurs, « il y a presque toujours, exprès, des malades du sida " (MLLDO, 120). Il s'agit, en l'occurrence, s'inscrivant dans la logique génocidaire qui prévaut dans l'univers diégétique de Murambi le livre des ossements, de mener l'extinction de la tribu des Tutsi puisque le premier rôle dévolu à la femme, dans la société, est la perpétuation de la race humaine ${ }^{13}$. Le cas du boucher de Murambi qui a préparé savamment et méticuleusement l'élimination de sa femme, une Tutsi, et de ses enfants, sortes de réceptacle des gènes Tutsi, en atteste. Il n'y a pas alors de langage adéquat pour décrire ce que l'on pourrait qualifier décemment de barbarie humaine ${ }^{14}$. Sinon comment décrire ces actes qui consistent à violer des femmes et de parachever cet acte horrible en « versant de l'acide dans le vagin [de la victime ou en lui enfonçant] des tessons de bouteille ou des morceaux de fer " (MLLDO, 121) ou des morceaux de bâton; ou encore de les tuer après les avoir violées " mille fois " (MLLDO, 119). Les violeurs joignent l'horreur à l'agréable. En témoigne l'exemple de cet Interahamwe (milicien Hutu) qui continue de

13 Bolya, avec la verve qu'on lui connaît, va plus loin dans un essai, au titre délibérément provocateur, La profanation des vagins - Le viol, arme de destruction massive, op. cit., et parle de " crime contre l'humanité ". On peut lire dans son résumé que "Cette profanation est bien une nouvelle arme biologique de guerre. Mode opératoire de cette barbarie, le viol devient un instrument de génocide, technique rustique d'extermination et de nettoyage ethnique ".

14 C'est devant le spectacle de toutes ces horreurs que Cornélius peut alors comprendre le propos qui, à ce moment-là, lui avait paru " cynique » et excessif, d'un célèbre intellectuel afro-américain. "Après son passage à Nyamata, complètement traumatisé, il avait déclaré à une chaîne de télévision : "Voilà, je me suis trompé toute ma vie. Après ce que j’ai vu au Rwanda, je pense que les nègres sont effectivement des sauvages. Je reconnais mon erreur. Je n'ai plus envie de me battre. Pour rien du tout ». Cornélius avait été très indigné en voyant ce monsieur pérorer avec un tel cynisme. Mais, à présent, il comprenait au moins pourquoi il avait perdu la tête ", Murambi le livre des ossements, 96. 
violer une femme agonisante alors qu'un de ses coreligionnaires vient de lui fracasser le crâne à coups de pierre. Au viol se greffe une dimension nécrophile car on voit bien la jouissance extatique que lui procure le corps duquel se retire la vie. En effet, il ne s'est pas « interrompu [et] a continué à besogner le corps agité de légers soubresauts. Il avait les yeux hors de la tête, tournés vers le ciel [pire même] il était encore plus excité qu'avant » (MLLDO, 222)

Ces violences prennent une dimension particulière lorsqu'elles sont commises par des victimes elles-mêmes que l'on oblige à s'instituer en leurs propres bourreaux. C'est le cas des mères que l'on contraint à piler leurs bébés avant qu'elles ne se fassent assassiner, des mères et des sœurs qui subissent des viols doublés d'un inceste de la part de parents, et tout cela, rien que pour le plaisir des miliciens Hutu. On le mesure aussi à des restes de femmes profanés qui disent toute cette violence, "l'ordre anormal des choses " (MLLDO, 144); des corps de femmes portant les stigmates de l'horreur et de la barbarie des hommes, des corps qui disent toute la souffrance et tous les supplices endurés. Le " corps conservé presque intact " (MLLDO, 96) de Theresa est exposé aux yeux des curieux qui ont, d'ailleurs, du mal à soutenir ce spectacle macabre. Cornélius en fit la triste expérience :

« La jeune femme avait la tête repoussée en arrière et le hurlement que lui avait arraché la douleur s'était figé sur son regard encore grimaçant. Ses magnifiques tresses étaient en désordre et ses jambes largement écartées. Un pieu - en bois ou en fer [...] - était resté encore enfoncé dans son vagin. » (MLLDO, 96)

Ce corps profané de Theresa qui gît parmi des milliers de cadavres dans le vaste cimetière qu'est devenue l'église témoigne des violences à la fois physiques et psychiques causées par le génocide. Cette violence faite au corps féminin s'observe également dans Allah n'est pas obligé même si les exemples ne sont pas nombreux, ils témoignent tout aussi de la barbarie qui sévit dans des univers en proie aux conflits. Les victimes y sont des jeunes filles, parfois à peine pubères, qui subissent des viols, quelque fois collectifs, et sont assassinées. Il en est ainsi, aussi, de celles qui servent de chairs fraîches à leurs compagnons de camp et aux chefs de guerre. Il faut dire que, généralement, elles représentent la part substantielle la plus agréable dans les butins de guerre.

Dans Murambi, le livre des ossements, par exemple, elles sont la part de gâteau du génocide que les jeunes mâles en rut Hutu se disputent 
(MLLDO, 111). Pour ces jeunes hommes, encore puceaux pour certains, ce génocide représente une occasion inespérée d'assouvir à loisir leurs instincts. Ces éphèbes savent combien "le chemin menant à l'intimité d'une femme est long, complexe et souvent décourageant " (MLLDO, 36). L'occasion de le trouver sans fournir d'effort leur est alors donnée avec le génocide. Et ils s'y adonnent avec entrain et sadisme. Ainsi, une fois repus, ils liquident leurs victimes (MLLDO, 108). Dans le roman de Kourouma, le narrateur s'arrête sur le cas de viol de quatre gamines : Fati, la fillette de sept ans, qui a été violée et assassinée (ANPO, p. 84); Sarah, une enfant de cinq ans, violée et laissée pour morte (ANPO, 96), qui se muera plus tard en bourreau lorsqu'elle deviendra une enfant-soldat; Sita, âgée de huit ans, dont le viol et le meurtre vont générer des expéditions punitives de la part de sœur Aminata qui veut venger sa mort (ANPO, p. 196); et Mirta, l'adolescente de douze ans, qui a subi un viol collectif (ANPO, 198). L'on comprend alors aisément que les personnes qui en ont la charge se transforment en lionnes pour les préserver, même s'il faut pour cela prendre les armes.

\section{DES « BOURREAUX » CONSACRÉS PAR LES ÉVÈNEMENTS : LES AGENTS}

Dans ce cadre, nous ferons une différence entre les dégradateurs, personnages qui influencent l'action (généralement négativement) et ceux que nous considérons comme de véritables amazones en ce sens qu'elles prennent et mènent des initiatives librement sans avoir à référer à quiconque si ce n'est à elles-mêmes. Pour les premiers, il s'agit de femmes qui participent quelque peu indirectement aux actions en les encourageant par leurs dires, ou encore celles qui se posent en complices passives par leur silence. S'agissant des secondes, ce sont des guerrières qui militent pour des causes qui leur sont chères et cela contre des hommes, fidèles en cela au mythe des amazones. Et comme nous l'avons déjà relevé plus haut, elles sont altruistes car leurs intérêts ne sont nullement égoïstes mais plutôt dignes de grands humanistes.

On ne rencontre pas d'adjuvants dans Allah n'est pas obligé au contraire de Murambi, le livre des ossements, qui campe des femmes Hutu dans le rôle de complices du génocide. C'est le cas de Rosa Karemera qui, souffrant de la présence de sa voisine Tutsi, la dénonce sans état d'âme à l'armée hutu sachant le sort qui lui sera réservé, alors que cette dernière a trouvé refuge parmi des Hutu. Sa soif de vengeance est aveugle à un point tel qu'elle se 
refuse à mesurer les risques qu'encourent les bonnes âmes (des Hutu) qui ont laissé parler leur cour en cachant « l'ennemie ». C'est aussi l'exemple de la mère de Faustin qui, bien que consciente du drame qui se joue, demeure " sereine " (MLLDO, 32) et silencieuse se contentant de se convaincre que c'est une histoire d'hommes. Louise, la sœur cadette de Faustin, elle, " est particulièrement fière " (MLLDO, 33) de ce que son fiancé fasse partie des troupes qui vont trucider les Tutsi. Quant à MarieHélène, l'épouse de Faustin, seule sa jalousie l'incite à s'assurer que son homme ne participera pas aux viols.

Toutes ces femmes acceptent et se font complices de meurtres, d'assassinats d'autres hommes dans une attitude plus ou moins passive et coupable. Elles n'ont aucune grandeur du fait qu'elles cautionnent des actes crapuleux et répréhensibles. D'autres, en revanche, s'insurgent contre les injustices et la violence et mettent toute leur énergie soit à rétablir un ordre pacifique, soit à protéger les plus faibles, même si pour cela elles doivent, parfois, faire appel à la violence comme celle qui leur est opposée. Ces dernières démentent le cliché sous entendu dans ce propos du père de Faustin dans Murambi, le livre des ossements qui, s'impatientant du retard pris par le génocide, se demande si les Tutsi les " prennent pour de vrais hommes ou pour des femmes? " (MLLDO, 25); ce sont des amazones.

Dans Murambi le livre des ossements, les seuls personnages qui campent dans le rôle de l'amazone sont Jessica et la sœur Félicité de par leur courage et leur abnégation dans la défense de leurs compatriotes. La religieuse Hutu est une sorte de martyr. Elle affronte la mort sans sourciller. Aux Interahamwe qui lui donnent une chance de sauver sa peau, par égard pour son frère, elle leur rétorque énergiquement, par deux fois, « Je veux mourir avec eux » (MLLDO, 141, 142). De fait, elle est consciente de ce que le droit de l'homme à la vie, ce droit le plus cher, n'appartient à aucun homme, conformément à ce que lui enseigne la morale chrétienne. Mais comme nous l'avons déjà signalé plus haut, le geste de cette femme est davantage lié à sa personne car, à aucun moment devant ses bourreaux, elle n'invoque des préceptes religieux pour plaider la cause de ses protégés. Le profond humanisme de sœur Félicité s'entr'aperçoit dans sa lettre d'adieu à son frère dans laquelle elle fait preuve de clémence à l'égard de ses assassins tout en les invitant à penser leur acte :

"Frère chéri, merci de vouloir maider. Mais au lieu de me sauver la vie et d'abandonner ceux dont j'ai la charge, les quarante-trois personnes, je choisis de mourir avec elles? Prie pour nous, que nous arrivions chez Dieu 
et dis au revoir à la vieille maman au frère. Je prierai pour toi, arrivée chez

Dieu. Porte-toi bien et merci de penser à moi " (MLLDO, 142)

Et il n'est pas alors étonnant que le milicien l'ait abattue d'un coup de pistolet en plein coeur comme pour détruire l'organe qui abritait autant d'amour pour l'homme, mais aussi celui qui lui renvoyait au visage ses propres manquements.

S'agissant de Jessica, c'est une patriote à l'image de ces combattants qui, sans tenir un fusil, agissent dans l'ombre. Le narrateur nous informe, en dressant son portrait que, très tôt (dès les premières luttes de son peuple), elle s'est fait militante. Elle a fait la guérilla avec les exigences que cela impose sans se défiler. Elle s'est également occupée des activités culturelles du FPR à Kigali et a participé aux négociations à Arusha. Pendant le génocide qui constitue la trame du roman, elle sert d'agent de liaison et assure des missions délicates et dangereuses qui l'amènent à risquer sa vie, tout simplement parce qu'elle a " choisi d'être là " (MLLDO, 43). Elle circule avec une fausse carte d'identité la désignant comme Hutu aux fins de mener à bien ces missions. Elle a dû, pour cela, vivre dans le mensonge, obligation imposée aux agents secrets. Il est des choses dont elle ne peut parler à personne même pas à sa meilleure amie Theresa qu'elle laissera ainsi aller se réfugier à l'église alors qu'elle sait qu'elle y sera massacrée : «C'est la situation qui le veut " (MLLDO, 37). Jessica n'a pu sauver cette amie chère à son cœur car, à cet instant, elle ne l'aurait pu. Il en est de même de la jeune femme qui requiert son aide pour se sauver des machettes des égorgeurs. Elle a ainsi acquis de la maturité et de l'expérience, paradoxalement, grâce à l'horreur. Cette femme singulière semble d'ailleurs être la seule, parmi tous les personnages ayant vécu le génocide, à avoir le moins accusé apparemment le coup des atrocités. On peut l'observer lorsqu'elle accompagne son ami Cornélius visiter les camps de l'horreur. Elle-même qualifie son engagement auprès des forces du FPR de "bon combat " (MLLDO, 84) car elle n'a jamais douté, "pas un seul instant » (MLLDO, p. 39) de leur victoire. C’est pourquoi, après la fin du génocide, elle continue sa lutte dans le bénévolat afin que de telles atrocités n'aient plus lieu.

Jessica est la narratrice première en termes de quantité du récit. C'est elle qui dit les horreurs du génocide, et ce, dans un langage simple. Il est d'ailleurs à relever qu'elle est la seule Tutsi que le récit représente dans le " combat ". Les actes et la participation des autres combattants se découvrent à travers son témoignage. Alors que ses amis, des hommes, qui ont vécu ces massacres se refusent à se souvenir (c'est le cas de Stanley), ou sont devenus fous ou muets devant l'indicible (comme Gérard le Matelot), 
elle est la seule à pouvoir en parler, à pouvoir y mettre des mots. C'est elle qui prend sur elle de révéler à Cornelius le rôle qu’a joué son père dans les massacres alors que tous les autres se dérobent se contentant de le regarder en chien de faïence.

Elle apparaît même, à certains moments, cynique. Il en est ainsi lorsqu'elle s'amuse des forfaits commis par ses compagnons de lutte contre leurs oppresseurs : "J'ai vu comment on les tirait comme des lapins" (MLLDO, 84). On pourrait alors croire qu'à l'instar des guerriers, seule importe l'issue du combat, à savoir être les vainqueurs et cela quel qu'en soit le prix. Et en pareille situation, les sentiments n'ont que peu de place. Puisque les vies des Tutsi n'ont que peu de prix, celles des Hutu n'en valent pas mieux; c'est le climat prévalant qui le commande. Mais Jessica se bat pour une cause juste et humaine, et son caractère déterminé l'y aide. Dans les moments de doute, elle se remémore le refrain scandé pendant les veillées d'armes avec ses compagnons de lutte: "Si les trois tombent au combat, les deux qui restent libéreront le Rwanda " (MLLDO, 46), et y puise l'énergie nécessaire pour repartir. L'image que ses amis, des hommes, ont d'elle c'est celle d'un vainqueur comme il en est dans le dessin la représentant en train de mimer le signe de la victoire. C'est elle qui annonce la prise de Kigali : "Kigali est définitivement entre nos mains " (MLLDO, 169). Le FPR s'efface derrière elle. Elle devient, par là même, un personnage englobant et, plus encore, un symbole qui représente tout un mouvement, toute une communauté.

C'est aussi pareille force de caractère que l'on retrouve chez les personnages de Kourouma dans Allah n'est pas obligé. Il y en a de deux sortes : les filles enfants-soldats et les femmes soldats. S'agissant des fillessoldats elles assument, bien évidemment, le rôle d'agents car elles sont membres de groupes armés et en ont tous les attributs, tels la tenue de parachutiste, des armes (des kalachnikovs). Et même si elles ne sont pas aussi fortes psychiquement que leurs pairs masculins, elles demeurent des soldats. Pour exemple, on a Sarah, devenue soldat par nécessité, et Fati qui se dopent au hasch qu'elles ne supportent manifestement pas. L'effet de ce psychotrope les rend " dingues ", en en croire le narrateur; ce qui les emmène à poser des actes irréfléchis. Sarah dans un de ses moments d'égarement trucidera son ami Tête Brûlée. Fati, elle, a "mitraillé » des jumeaux avec sa kalachnikov alors qu'elle "était dans les vapeurs "(ANPO, 98). Toutes les deux participent aux expéditions menées par leur troupe et comptent parmi les plus redoutables et les plus cruelles.

Parmi les femmes-soldats, il faut aussi établir une distinction entre celles qui portent des armes sans vraiment s'en servir et les autres qui en ont 
et s'en servent. Le général Onika et ses brus, dans Allah n'est pas obligé, font partie des premières. Si elles possèdent les grades et les tenues qui en sont les attributs (galons et armes), elles sont davantage des donneuses d'ordre. Elles s'occupent, en particulier, de gagner des dollars et de les préserver, et ce sont les soldats qu'elles commandent qui mènent les combats. La preuve est qu'elles ne maîtrisent pas l'art de la guerre. Cela se donne à voir lorsque Saniquellie, le fief du général Onika Baclay, essuie des attaques que ses soldats n'arrivent ni à contenir, ni à repousser du fait de son inefficacité à prendre des décisions appropriées en pareille situation. Le général préfere s'en remettre entièrement aux féticheurs et marabouts qui lui assurent une victoire certaine sur ses ennemis ${ }^{15}$. Et elle le paiera lourdement d'abord avec la perte de nombreux enfants-soldats lors des combats et, ensuite, avec la prise de Saniquellie avec toutes ses richesses par les forces du NPLF alors qu'elle a pris le parti de se déplacer avec tout son état-major abandonnant son territoire, sans gardes.

Pour les guerrières que sont sœur Marie-Béatrice et sœur Hadja Gabrielle Aminata, elles commandent des brigades spéciales dans des camps retranchés avec une discipline de fer. La première montrera tout ce dont elle est capable dans des affrontements contre une bande de brigands et le redoutable chef de guerre, le Prince Johnson, qui veulent s'emparer de son institution. Cette femme devient une combattante, par devers elle, sans entraînement préalable et sans que rien ni personne (pas même elle), ne l'ait prévu. Alors que les forces de garde affectées à son service par l'évêché, dès le déclenchement de la guerre, décampent au pas de course devant les attaques de Johnson et de ses pillards, la nonne prend les choses en main :

"Marie-Béatrice se fâcha, se débarrassa de la cornette, arracha de la main d'un soldat un kalach. Et se coucha. Et mitrailla et mitrailla. Cinq pillards furent fauchés et les autres détalèrent, détalèrent sans demander leur reste; à partir de là sainte Marie-Béatrice prit en main, d'une main de fer, la défense de l'institution. Elle signifia au capitaine que lui et tous ses hommes devaient obéir à elle et à elle seule " (ANPO, 147).

On ne peut manquer de relever, dans l'extrait sus cité, la récurrence des verbes d'action, la gradation ascendante dans les gestes et surtout les figures d'insistance (l'épanalepse, l'anadiplose, l'épanaphore) qui en rajoutent à la

15 Il faut aussi lire dans cette position, l'ironie du narrateur qui reprend là, le cliché qui veut que les femmes soient les clientes privilégiées des voyants, féticheurs et autres diseurs de bonne aventure. 
magnitude du personnage qu'accentue l'attitude des poltrons qui lui font face. La scène ne manque pas d'humour. Elle résistera ainsi, quatre mois durant, aux pillards, ce qui, insiste le narrateur, tient tout simplement du miracle. Le narrateur, d'ailleurs, se rit des hommes qu'elle ridiculise en les surprenant par sa hargne, son courage et sa perspicacité. C'est le cas lorsque, par trois fois, elle repoussera les assauts de Prince Johnson arme au poing. À chaque fois, même à des heures indues, alors qu'il espérait la prendre en traître, "il n'y a pas eu de surprise! La sainte était informée " (ANPO, 156). Mal en a pris donc le chef de guerre qui, sûr de lui, " avait cru que c'était une simple balade pour ses enfants-soldats" (ANPO, 156). Il en sera marqué au point qu'il proposera à la sœur Marie-Béatrice le poste de chef de sa brigade féminine, quand il put la soumettre. La logique veut aussi qu'il ait pensé qu'il valait mieux l'avoir avec lui que contre lui. Il en fit même sa maîtresse s'assurant ainsi, profondément, de sa loyauté.

Sour Hadja Gabrielle Aminata, elle, est encore plus vindicative que sa consœur. À la différence de Marie-Béatrice, elle aime les armes au maniement desquels elle s'initie ainsi que ses protégés. C'est " une garce de matrone à la mitraillette rapide " (ANPO, 194) qui commande une brigade spéciale de filles dans une cité que l'on pourrait assimiler à un village d'amazones. A Mile-Thirty-Eight, une sorte de camp retranché, vivent des femmes et des jeunes filles impressionnantes dans l'art de la guerre. À leur manière d'agir on croirait avoir affaire à des soldats d'une armée secrète à l'image de la fameuse légion étrangère française dont les membres peuvent être tenus, à certains égards, pour des sortes de mercenaires impitoyables. On les voit à l'œuvre lorsqu'elles vont mener des expéditions punitives pour venger la mort d'une des leurs, Sita, qui a été sauvagement assassinée par des pervers qu'elles punirent à la mesure du crime qu'ils ont commis. Elles semèrent la terreur en tuant, émasculant et décapitant des travailleurs des campements voisins qui osaient s'en éloigner dans la nuit. Elles réitéreront ces actes quand Mirta sera violée collectivement par des chasseurs. Et c'est en « hérö̈ne de guerre » morte sur le champ de bataille que Hadja Gabrielle sera célébrée par ses ennemis, les chasseurs, qui l'honorèrent conformément à leur code d'honneur, témoignant parallèlement leur admiration devant de sa grandeur.

Toutes ces femmes ont confirmé ce qu'André Malraux affirmait déjà dans L'espoir à savoir qu' "Il n'y a pas cinquante manières de combattre, il n'y en a qu'une, c'est d'être vainqueur. Ni la révolution, ni la guerre ne consistent à se plaire à soi-même ${ }^{16}$. Et victorieuses elles l'ont été

16 André Malraux, L’espoir, Paris, Gallimard, $2^{\mathrm{e}}$ partie, II, 12. 
assurément en prenant sur elles de mener des combats nobles, pour une cause juste : l'homme plus que jamais au centre de toutes les préoccupations du monde actuel.

\section{Conclusion}

Boubacar Boris Diop et Ahmadou Kourouma ont choisi de dire dans Murambi, le livre des ossements et Allah n'est pas obligé, des histoires de femmes dans des situations de conflits. Ces dernières sont loin d'être commodes déjà pour les hommes qui, pourtant, ne réservent pas de sort enviable aux femmes qu'ils tiennent pour des êtres faibles. Conscientes de cela, elles se chargent toutes seules d'assurer leur protection pour celles qui le peuvent. Leur implication est des plus éloquentes car elle signifie que chaque être a une place dans la société et qu'il est préférable de n'assigner, au préalable, à quiconque (femme ou homme) un espace déterminé ou encore un rôle établi, comme il en est depuis l'aube des temps. Les exemples répertoriés en analyse montrent, en définitive, que ce sont les situations qui définissent les manières d'être, de penser et d'agir des individus en société qui sont, souvent, loin d'obéir au construit social et historique de l'appréhension des genres.

\section{Bibliographie}

Amossy Ruth, Les idées reçues. Sémiologie du stéréotype, Paris, Nathan, 1991. Bourdieu Pierre, la domination masculine, Paris, Seuil, 1998.

Bremond Claude, Logique du récit, Paris, Seuil, 1973.

Diop Boubacar Boris, Murambi le livre des ossements, Paris, Stock, 2001. Greimas Algirdas Julien, Sémantique structurale, Paris, PUF, 1986, Rééd. Kourouma Ahmadou, Allah n'est pas obligé, Paris, Seuil, 2001. 